\title{
Characteristics of physical properties in soil profiles under selected introduced trees in the Nature Reserve Arboretum Mlyňany, Slovakia
}

\author{
Nora Polláková ${ }^{*}$, Vladimír Šimanský1, Jerzy Jonczak ${ }^{2}$ \\ ${ }^{1}$ Department of Soil Science and Geology, Slovak University of Agriculture in Nitra, Tr. A. Hlinku 2, 94976 \\ Nitra, Slovak Republic \\ ${ }^{2}$ Department of Soil Environment Sciences, Warsaw University of Life Sciences, Nowoursynowska Str. 159, \\ 02-776 Warsaw, Poland
}

\begin{abstract}
Polláková, N., Šimanský, V., JoncZak, J., 2017. Characteristics of physical properties in soil profiles under selected introduced trees in the Nature Reserve Arboretum Mlyňany, Slovakia. Folia Oecologica, 44: 78-86.

The relationship between introduced trees roots and soils in which they grow is the most important factor influencing the adaptation, growth and health of these trees. Therefore, the objective of this study was to identify which physical soil properties enhance or limit the vitality of the studied introduced trees in the Arboretum Mlyňany. Soil properties were studied in seven soil profiles under dense monocultures of Chamaecyparis lawsoniana, Liriodendron tulipifera, Juniperus Chinensis, Thuja orientalis, Thuja plicata, Picea orientalis and Pinus nigra. The results showed that all stagnic horizons had exceeded the limit values of total porosity and bulk density, hence these horizons were compacted. Based on the soil and climatic requirements of the examined trees we conclude that the soil properties of their sites in arboretum are suitable for: Chamaecyparis lawsoniana, Liriodendron tulipifera, Thuja orientalis and Pinus nigra. Nevertheless, physical properties in profiles under Picea orientalis and Juniperus Chinensis do not permit rapid drainage of water, what is unfavourable for healthy development of these two species; while Thuja plicata demanding high moisture supply is grown on soil with high coarse porosity, a prerequisite of fast rainwater drainage. However, since none of the studied introduced trees had suffered from physiological disorders or diseases, they may be declared acclimatized well in the soil-climate conditions described in this study.
\end{abstract}

\section{Keywords}

acclimatization, compaction, introduced trees, soil aeration, soil moisture, soil porosity

\section{Introduction}

The Arboretum Mlyňany (Slovakia) was established in year 1892 by Count Ambrózy with the main goal to collect introduced sempervirent tree species. The acclimatization of hundreds of non-native, introduced taxa has been studied mostly for their use as ornamentals. The recent inventory of the gene pool of trees and shrubs showed that the current number of taxa grown in the Arboretum is 1,933 (HoŤKA et al., 2013). Introduction of species involves adaptation, productivity and success in new types of environmental conditions. Since invasion is considered as the highest level of successful introduction, species with invasive behaviour should be excluded from intentional introduction (ELiášs, 2011).

In general, each plant species has specific requirements for soil-ecological environment. If plants are to grow to their potential, they must be provided by a satisfactory soil environment. On the other hand, inappropriate conditions may limit or even make it impossi-

*Corresponding author: e-mail: nora.pollakova@uniag.sk 
ble to grow a given plant species. Tree growth requires adequate availability of water and oxygen from the soil as well as a sufficient supply of nutrients, light and heat (WALl and HeisKanen, 2009). Many works define mainly chemical and biological soil properties affecting plants growth. The physical properties are considerably undervalued, despite the fact that excessively wet or dry, shallow or impermeable soils can severely limit or even interrupt the growth of plants (HUXLEY et al., 1992).

For favourable course of biological processes as well as the life of soil organisms and plant roots, it is important to provide a sufficient supply of water and air into the soil. On the base of soil moisture monitoring, which is carried out in Slovakia since 1970, it can be stated that in the recent years there were significant changes in the dynamics of soil moisture, available water supplies and soil moisture stratification. In contrast to mountain forests, poor moisture conditions are in the lowest forest zones, in the areas where output of water significantly exceeds atmospheric precipitation, and the ability of soil to provide enough utilizable water usually covers only the first half of growing season. Unfavourable moisture is one of the main causes deteriorating soil environment with tendency of physiological weakening and even necrosis of trees (TUŽINSKÝ, 2007). Soil moisture not only affects physical, chemical and biological soil properties, but it is also essential for plant growth. The amount of soil water used by plant varies depending on characteristics of soil e.g., texture and plant e.g., roots distribution, depth and transpiration coefficient (Hosseinia et al., 2016).

Since favourable rooting space, abundance of nutrients, water and appropriate air exchange in the soil are important conditions for right tree life, the aim of the study was to find out which physical soil properties promote or limit the vitality of the studied introduced tree species in the Arboretum Mlyňany.

\section{Materials and methods}

\section{Study site}

Arboretum Mlyňany ( $48^{\circ} 19^{\prime} \mathrm{N}$, and $\left.18^{\circ} 21^{\prime} \mathrm{E}\right)$ is located in southern Slovakia, in the valley of the Žitava River, at an altitude of 165-217 $\mathrm{m}$ above the sea level, with a mean annual temperature of $10.6{ }^{\circ} \mathrm{C}$ and a mean annual total precipitation of $541 \mathrm{~mm}$ (HRUBíK et al., 2011). Undulated terrain is southern spur of the Hronský Inovec and Tribeč hills. The Arboretum site floristically belongs to the Pannonian area, geo-botanically to the Querceto-Carpinteum area. It is situated on a late Tertiary geological formation, represented by clays, sands and rubble sands (STENHÜBEL, 1957). This substratum is almost all covered by wind-deposited loess, mostly without carbonates. Neogene gravels, patchily located closer to the soil surface, cause significant deteriora- tion of soil conditions, since they are highly permeable and poor in nutrients (CIFRA, 1958). On majority of the arboretum area Stagni-Haplic Luvisols have been developed (Polláková, 2013). For coherent impression of vegetation, different tree species were planted in monocultures. Nowadays, the Arboretum is composed of: original Ambrozy's park founded in year 1892, area of East-Asian dendroflora in year 1964, area of North American dendroflora in year 1975, area of Korean dendroflora in year 1984 and exposition of Slovak dendroflora founded in year 1992 (TÁBOR and PAVLAČKA, 1992).

\section{Soil sampling and analyses}

Soil properties were studied in seven sites under dense monocultures of introduced trees. The area of each chosen tree species site was at least $200 \mathrm{~m}^{2}$. Since the Arboretum Mlyňany is a Nature Reserve, our opportunity for digging soil pits was limited. Therefore, for each examined tree species, we dug one soil pit roughly in the middle of its monoculture.

Soil pits in the original Ambrózy park founded in 1892 in an oak-hornbeam forest:

- under 100-year-old western redcedars (Thuja plicata, Donn ex D. Don)

- under 110-years-old oriental spruces (Picea orientalis, L.)

- under 110-years-old Austrian pines (Pinus nigra, Arnold).

Soil pits in the East Asian area established in 1964 on land that was until 1960 used as arable:

- under 50-years-old oriental thujas (Thuja orientalis, L.)

- under 50-years-old Chinese junipers (Juniperus Chinensis, L.).

Soil pits in the North American area founded in 1975 on land that was previously used as vineyard:

- under 40-years-old Lawson cypresses (Chamaecyparis lawsoniana, Murr.)

- under 40-years-old tulip trees (Liriodendron tulipifera, L.).

In spring 2015, undisturbed soil samples $\left(100 \mathrm{~cm}^{3}\right)$ were collected using steel cylinders per each of $10 \mathrm{~cm}$ layer to the depth of 80 and $90 \mathrm{~cm}$, in three repeats. In the samples there were determined physical (particle density, bulk density, porosity, coarse and capillary pores) and hydro-physical soil properties (soil aeration, air-filled porosity, maximal capillary water capacity, available water, available water capacity and wilting point) according to the standard methods (HRIVŇÁKOvÁ et al., 2011). Textural composition was determined for all soil horizons of each profile, using the pipette method (HrivŇÁKoví et al., 2011).

All analyses of physical properties were done in three repeats and this study shows the average values (mean $\pm \mathrm{SD})$. 


\section{Results and discussion}

Bulk density and porosity of soil profiles in relation to studied tree species

In all the studied profiles, with increased depth there almost equally increased values of reduced bulk density $\left(\rho_{\mathrm{d}}\right)$ and decreased total porosity $\left(\mathrm{P}_{\mathrm{T}}\right)$, mainly in luvic (lv) and stagnic (st) horizons enriched by clay (Table 1). Generally, soils containing high percentage of clay are prone to compaction (MATI et al., 2011; SAFADOUST et al., 2014). According to the limit values of $\rho_{d}$ and $P_{T}$ proposed by LHOTsKÝ et al. (1984) for different textural groups, in the studied profiles of the Arboretum, all st horizons and beside tulip trees and oriental spruces also lv horizons, exceeded the limit values of total porosity and bulk density, hence these horizons were compacted. Following the limit values of LHOTSKÝ et al. (1984), the $\rho_{\mathrm{d}}$ higher than $1.40 \mathrm{t} \mathrm{m}^{-3}$ and $\mathrm{P}_{\mathrm{T}}$ below $47 \%$ in clayey loam (IH); $\rho_{\mathrm{d}}>1.45 \mathrm{t} \mathrm{m}^{-3}$ and $\mathrm{P}_{\mathrm{T}}<45 \%$ in loam $(\mathrm{H})$; $\rho_{\mathrm{d}}>1.55 \mathrm{t} \mathrm{m}^{-3}$ and $\mathrm{P}_{\mathrm{T}}<42 \%$ in sandy loam (PH); $\rho_{\mathrm{d}}>$ $1.60 \mathrm{t} \mathrm{m}^{-3}$ and $\mathrm{P}_{\mathrm{T}}<40 \%$ in loamy sand (HP) are critical because plant roots barely grow through the soil. Moreover, DeXTER (2004) stated that soil compaction has negative effect on plant root growth through reducing the storage and supply of water and nutrients. Consequently, it causes decreased growth (biomass) responses of the whole plant and of the plant's compo- nents such as stem, shoot, leaves, roots (JOURGHOLAMI et al., 2016). Adverse effect of soil compaction mainly occurs through increasing soil bulk density and soil strength, decreasing macro porosity, soil water infiltration and water-holding capacity, what may negatively influence soil biological properties (FREY et al., 2009). In profile under Austrian pines, exceeding critical $\rho_{d}$ and $\mathrm{P}_{\mathrm{T}}$ in fragic ( $\mathrm{fg}$ ) horizon was caused by high content of gravel $(30 \%)$ and sand $(65 \%)$. However, the volume of air-filled porosity $\left(\mathrm{V}_{\mathrm{A}} 21.6 \%\right)$, coarse pores $\left(\mathrm{P}_{\mathrm{N}}\right.$ $20.2 \%$ ) was relatively high, even higher than the fine, capillary pores $\left(\mathrm{P}_{\mathrm{C}} 15.6 \%\right)$. Comparing these values with $20 \%$ volume of macropores and air-filled porosity favourable for trees growth suggested by WALL and HeISKANEN (2003), the fg horizon under Austrian pines was not considered as compacted (Tables 1-2).

High volumes of coarse pores in the upper soil layers under Chinese junipers, western redcedars, oriental spruces and Austrian pines highlight good drainage of these soils (Table 1, Fig. 1). However, rapid drainage of rainwater can negatively influence trees by lack of water, mainly during long summer periods of drought, which are according to TUŽINSKÝ (2007) typical for this region. In turn, higher clay content in lv and st horizons was accompanied by higher percentage (28-37\%) of fine, capillary pores retaining water. Thus, majority of rainwater retain in soil profiles, and does not drain to deeper layers.
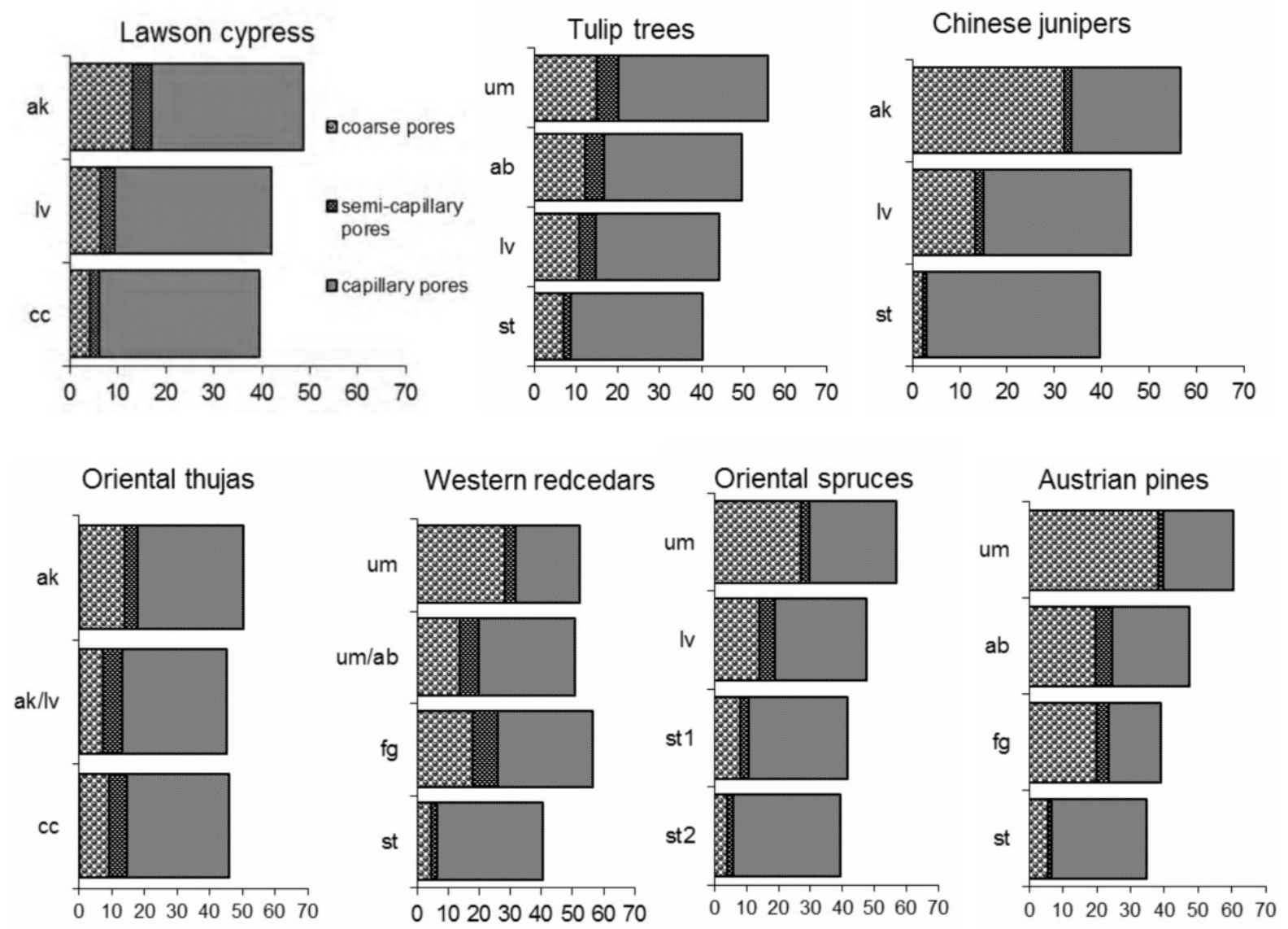

Fig. 1. Distribution of coarse, semi-capillary and capillary pores in studied soil profiles (in \%). 
Table 1. Mean ( \pm standard deviation) soil physical properties in profiles and soil texture

\begin{tabular}{|c|c|c|c|c|c|c|c|c|c|c|}
\hline $\begin{array}{c}\text { Tree } \\
\text { species }\end{array}$ & Horizon & $\begin{array}{l}\text { Depth } \\
(\mathrm{m})\end{array}$ & $\begin{array}{c}\text { Texture } \\
(\Delta)\end{array}$ & $\begin{array}{l}\text { Texture } \\
\text { (Novák) }\end{array}$ & \multicolumn{2}{|c|}{$\left(\mathrm{t} \mathrm{m}^{-3}\right)$} & $\mathrm{P}_{\mathrm{T}}$ & $\begin{array}{l}P_{N} \\
(\%)\end{array}$ & $\mathrm{P}_{\mathrm{C}}$ & $\mathrm{PS}_{\mathrm{S}}$ \\
\hline \multirow{3}{*}{$\begin{array}{l}\text { Lawson } \\
\text { cypress }\end{array}$} & ak & $0-30$ & ssh & $\mathrm{H}$ & $\begin{array}{c}2.57 \\
( \pm 0.04)\end{array}$ & $\begin{array}{c}1.31 \\
( \pm 0.15)\end{array}$ & $\begin{array}{c}48.9 \\
( \pm 5.3)\end{array}$ & $\begin{array}{c}13.0 \\
( \pm 5.2)\end{array}$ & $\begin{array}{c}31.7 \\
( \pm 1.6)\end{array}$ & $\begin{array}{c}4.2 \\
( \pm 1.0)\end{array}$ \\
\hline & lv & $30-70$ & si & $\mathrm{H}$ & $\begin{array}{c}2.60 \\
( \pm 0.02)\end{array}$ & $\begin{array}{c}1.51 \\
( \pm 0.04)\end{array}$ & $\begin{array}{c}42.1 \\
( \pm 1.5)\end{array}$ & $\begin{array}{c}6.5 \\
( \pm 1.8)\end{array}$ & $\begin{array}{c}32.6 \\
( \pm 1.0)\end{array}$ & $\begin{array}{c}3.0 \\
( \pm 0.7)\end{array}$ \\
\hline & $\mathrm{cc}$ & $70-80$ & sh & $\mathrm{H}$ & $\begin{array}{c}2.61 \\
( \pm 0.01)\end{array}$ & $\begin{array}{c}1.58 \\
( \pm 0.02)\end{array}$ & $\begin{array}{c}39.6 \\
( \pm 1.2)\end{array}$ & $\begin{array}{c}4.1 \\
( \pm 1.1)\end{array}$ & $\begin{array}{c}33.3 \\
( \pm 0.9)\end{array}$ & $\begin{array}{c}2.2 \\
( \pm 0.1)\end{array}$ \\
\hline \multirow{4}{*}{ Tulip trees } & um & $0-20$ & ssh & $\mathrm{H}$ & $\begin{array}{c}2.54 \\
( \pm 0.04)\end{array}$ & $\begin{array}{c}1.12 \\
( \pm 0.03)\end{array}$ & $\begin{array}{c}55.9 \\
( \pm 1.2)\end{array}$ & $\begin{array}{c}15.1 \\
( \pm 2.7)\end{array}$ & $\begin{array}{c}35.6 \\
( \pm 1.9)\end{array}$ & $\begin{array}{c}5.2 \\
( \pm 0.2)\end{array}$ \\
\hline & $a b$ & $20-50$ & $\mathrm{sp}$ & $\mathrm{PH}$ & $\begin{array}{c}2.58 \\
( \pm 0.00)\end{array}$ & $\begin{array}{c}1.30 \\
( \pm 0.09)\end{array}$ & $\begin{array}{c}49.6 \\
( \pm 3.7)\end{array}$ & $\begin{array}{c}12.1 \\
( \pm 1.9)\end{array}$ & $\begin{array}{c}32.9 \\
( \pm 2.1)\end{array}$ & $\begin{array}{c}4.6 \\
( \pm 0.9)\end{array}$ \\
\hline & lv & $50-70$ & sh & $\mathrm{PH}$ & $\begin{array}{c}2.59 \\
( \pm 0.01)\end{array}$ & $\begin{array}{c}1.44 \\
( \pm 0.08)\end{array}$ & $\begin{array}{c}44.3 \\
( \pm 3.2)\end{array}$ & $\begin{array}{c}10.7 \\
( \pm 3.3)\end{array}$ & $\begin{array}{c}29.4 \\
( \pm 0.8)\end{array}$ & $\begin{array}{c}4.2 \\
( \pm 0.2)\end{array}$ \\
\hline & st & $70-90$ & si & $\mathrm{H}$ & $\begin{array}{c}2.59 \\
( \pm 0.01)\end{array}$ & $\begin{array}{c}1.54 \\
( \pm 0.03)\end{array}$ & $\begin{array}{c}40.3 \\
( \pm 1.0)\end{array}$ & $\begin{array}{c}7.2 \\
( \pm 0.7)\end{array}$ & $\begin{array}{c}31.5 \\
( \pm 0.9)\end{array}$ & $\begin{array}{c}1.6 \\
( \pm 0.6)\end{array}$ \\
\hline \multirow{3}{*}{$\begin{array}{l}\text { Chinese } \\
\text { junipers }\end{array}$} & ak & $0-20$ & sh & $\mathrm{H}$ & $\begin{array}{c}2.55 \\
( \pm 0.09)\end{array}$ & $\begin{array}{c}1.11 \\
( \pm 0.21)\end{array}$ & $\begin{array}{c}56.5 \\
( \pm 8.9)\end{array}$ & $\begin{array}{c}32.0 \\
( \pm 9.9)\end{array}$ & $\begin{array}{c}23.1 \\
( \pm 2.7)\end{array}$ & $\begin{array}{c}1.4 \\
( \pm 0.4)\end{array}$ \\
\hline & lv & $20-30$ & ts & $\mathrm{IH}$ & $\begin{array}{c}2.61 \\
( \pm 0.00)\end{array}$ & $\begin{array}{c}1.41 \\
( \pm 0.03)\end{array}$ & $\begin{array}{c}45.9 \\
( \pm 1.0)\end{array}$ & $\begin{array}{c}13.3 \\
( \pm 4.9)\end{array}$ & $\begin{array}{c}31.0 \\
( \pm 5.0)\end{array}$ & $\begin{array}{c}1.6 \\
( \pm 0.1)\end{array}$ \\
\hline & st & $30-80$ & $\mathrm{ti}$ & IH & $\begin{array}{c}2.58 \\
( \pm 0.01)\end{array}$ & $\begin{array}{c}1.56 \\
( \pm 0.03)\end{array}$ & $\begin{array}{c}39.5 \\
( \pm 1.0)\end{array}$ & $\begin{array}{c}2.2 \\
( \pm 1.1)\end{array}$ & $\begin{array}{c}36.6 \\
( \pm 2.1)\end{array}$ & $\begin{array}{c}0.7 \\
( \pm 0.2)\end{array}$ \\
\hline \multirow{3}{*}{$\begin{array}{l}\text { Oriental } \\
\text { thujas }\end{array}$} & ak & $0-20$ & si & $\mathrm{H}$ & $\begin{array}{c}2.61 \\
( \pm 0.02)\end{array}$ & $\begin{array}{c}1.30 \\
( \pm 0.17)\end{array}$ & $\begin{array}{c}50.2 \\
( \pm 6.2)\end{array}$ & $\begin{array}{c}13.7 \\
( \pm 4.8)\end{array}$ & $\begin{array}{c}32.3 \\
( \pm 0.6)\end{array}$ & $\begin{array}{c}4.2 \\
( \pm 0.9)\end{array}$ \\
\hline & $\mathrm{ak} / \mathrm{lv}$ & $20-40$ & ssi & $\mathrm{H}$ & $\begin{array}{c}2.61 \\
( \pm 0.03)\end{array}$ & $\begin{array}{c}1.43 \\
( \pm 0.12)\end{array}$ & $\begin{array}{c}45.2 \\
( \pm 4.3)\end{array}$ & $\begin{array}{c}7.4 \\
( \pm 2.4)\end{array}$ & $\begin{array}{c}32.1 \\
( \pm 1.8)\end{array}$ & $\begin{array}{c}5.7 \\
( \pm 2.1)\end{array}$ \\
\hline & $\mathrm{cc}$ & $40-80$ & ssh & $\mathrm{H}$ & $\begin{array}{c}2.63 \\
( \pm 0.03)\end{array}$ & $\begin{array}{c}1.42 \\
( \pm 0.03)\end{array}$ & $\begin{array}{c}45.9 \\
( \pm 1.3)\end{array}$ & $\begin{array}{c}9.1 \\
( \pm 1.7)\end{array}$ & $\begin{array}{c}31.3 \\
( \pm 0.2)\end{array}$ & $\begin{array}{c}5.5 \\
( \pm 1.1)\end{array}$ \\
\hline \multirow{4}{*}{$\begin{array}{l}\text { Western } \\
\text { redcedars }\end{array}$} & um & $0-10$ & sh & $\mathrm{H}$ & $\begin{array}{c}2.54 \\
( \pm 0.01)\end{array}$ & $\begin{array}{c}1.15 \\
( \pm 0.08)\end{array}$ & $\begin{array}{c}54.5 \\
( \pm 3.2)\end{array}$ & $\begin{array}{c}25.3 \\
( \pm 3.9)\end{array}$ & $\begin{array}{c}25.7 \\
( \pm 1.8)\end{array}$ & $\begin{array}{c}3.6 \\
( \pm 0.2)\end{array}$ \\
\hline & $\mathrm{um} / \mathrm{ab}$ & $10-22$ & sh & $\mathrm{H}$ & $\begin{array}{c}2.52 \\
( \pm 0.00)\end{array}$ & $\begin{array}{c}1.24 \\
( \pm 0.05)\end{array}$ & $\begin{array}{c}50.8 \\
( \pm 1.9)\end{array}$ & $\begin{array}{c}13.8 \\
( \pm 4.5)\end{array}$ & $\begin{array}{c}31.0 \\
( \pm 4.0)\end{array}$ & $\begin{array}{c}6.0 \\
( \pm 0.1)\end{array}$ \\
\hline & $\mathrm{fg}$ & $22-50$ & sh & $\mathrm{H}$ & $\begin{array}{c}2.56 \\
( \pm 0.02)\end{array}$ & $\begin{array}{c}1.11 \\
( \pm 0.09)\end{array}$ & $\begin{array}{c}56.6 \\
( \pm 3.8)\end{array}$ & $\begin{array}{c}18.1 \\
( \pm 2.5)\end{array}$ & $\begin{array}{c}30.6 \\
( \pm 2.7)\end{array}$ & $\begin{array}{c}7.9 \\
( \pm 0.2)\end{array}$ \\
\hline & st & $50-80$ & $\mathrm{ti}$ & $\mathrm{IH}$ & $\begin{array}{c}2.55 \\
( \pm 0.01)\end{array}$ & $\begin{array}{c}1.51 \\
( \pm 0.04)\end{array}$ & $\begin{array}{c}40.6 \\
( \pm 1.7)\end{array}$ & $\begin{array}{c}4.7 \\
( \pm 1.4)\end{array}$ & $\begin{array}{c}34.2 \\
( \pm 1.2)\end{array}$ & $\begin{array}{c}1.7 \\
( \pm 0.6)\end{array}$ \\
\hline \multirow{4}{*}{$\begin{array}{l}\text { Oriental } \\
\text { spruces }\end{array}$} & um & $0-20$ & ssh & PH & $\begin{array}{c}2.54 \\
( \pm 0.04)\end{array}$ & $\begin{array}{c}1.09 \\
( \pm 0.09)\end{array}$ & $\begin{array}{c}57.1 \\
( \pm 3.3)\end{array}$ & $\begin{array}{c}27.1 \\
( \pm 6.0)\end{array}$ & $\begin{array}{c}27.3 \\
( \pm 3.5)\end{array}$ & $\begin{array}{c}2.7 \\
( \pm 0.1)\end{array}$ \\
\hline & lv & $20-40$ & ssi & $\mathrm{H}$ & $\begin{array}{c}2.58 \\
( \pm 0.01)\end{array}$ & $\begin{array}{c}1.35 \\
( \pm 0.05)\end{array}$ & $\begin{array}{c}47.4 \\
( \pm 2.1)\end{array}$ & $\begin{array}{c}14.3 \\
( \pm 2.4)\end{array}$ & $\begin{array}{c}28.5 \\
( \pm 0.7)\end{array}$ & $\begin{array}{c}4.6 \\
( \pm 0.2)\end{array}$ \\
\hline & $\mathrm{st}_{1}$ & $40-62$ & si & $\mathrm{H}$ & $\begin{array}{c}2.55 \\
( \pm 0.01)\end{array}$ & $\begin{array}{c}1.49 \\
( \pm 0.03)\end{array}$ & $\begin{array}{c}41.5 \\
( \pm 1.3)\end{array}$ & $\begin{array}{c}8.2 \\
( \pm 2.0)\end{array}$ & $\begin{array}{c}30.6 \\
( \pm 2.1)\end{array}$ & $\begin{array}{c}2.7 \\
( \pm 0.1)\end{array}$ \\
\hline & $\mathrm{st}_{2}$ & $62-80$ & $\mathrm{ti}$ & $\mathrm{IH}$ & $\begin{array}{c}2.54 \\
( \pm 0.00)\end{array}$ & $\begin{array}{c}1.54 \\
( \pm 0.02)\end{array}$ & $\begin{array}{c}39.4 \\
( \pm 0.9)\end{array}$ & $\begin{array}{c}4.1 \\
( \pm 1.6)\end{array}$ & $\begin{array}{c}33.5 \\
( \pm 2.8)\end{array}$ & $\begin{array}{c}1.8 \\
( \pm 0.1)\end{array}$ \\
\hline \multirow{4}{*}{$\begin{array}{l}\text { Austrian } \\
\text { pines }\end{array}$} & um & $0-10$ & $\mathrm{sp}$ & $\mathrm{PH}$ & $\begin{array}{c}2.55 \\
( \pm 0.00)\end{array}$ & $\begin{array}{c}1.01 \\
( \pm 0.06)\end{array}$ & $\begin{array}{c}60.5 \\
( \pm 2.2)\end{array}$ & $\begin{array}{c}38.4 \\
( \pm 3.4)\end{array}$ & $\begin{array}{c}20.7 \\
( \pm 1.1)\end{array}$ & $\begin{array}{c}1.4 \\
( \pm 0.1)\end{array}$ \\
\hline & $a b$ & $10-38$ & $\mathrm{sp}$ & HP & $\begin{array}{c}2.62 \\
( \pm 0.05)\end{array}$ & $\begin{array}{c}1.38 \\
( \pm 0.06)\end{array}$ & $\begin{array}{c}47.5 \\
( \pm 2.3)\end{array}$ & $\begin{array}{c}19.7 \\
( \pm 3.5)\end{array}$ & $\begin{array}{c}23.0 \\
( \pm 2.9)\end{array}$ & $\begin{array}{c}4.8 \\
( \pm 0.2)\end{array}$ \\
\hline & fg & $38-70$ & $\mathrm{sp}$ & $\mathrm{H}$ & $\begin{array}{c}2.66 \\
( \pm 0.04)\end{array}$ & $\begin{array}{c}1.62 \\
( \pm 0.06)\end{array}$ & $\begin{array}{c}39.1 \\
( \pm 2.9)\end{array}$ & $\begin{array}{c}20.2 \\
( \pm 2.6)\end{array}$ & $\begin{array}{c}15.6 \\
( \pm 2.6)\end{array}$ & $\begin{array}{c}3.3 \\
( \pm 0.4)\end{array}$ \\
\hline & st & 70-90 & tp & $\mathrm{H}$ & $\begin{array}{c}2.60 \\
( \pm 0.01)\end{array}$ & $\begin{array}{c}1.70 \\
( \pm 0.04)\end{array}$ & $\begin{array}{c}34.7 \\
( \pm 1.5)\end{array}$ & $\begin{array}{c}5.7 \\
( \pm 1.3)\end{array}$ & $\begin{array}{c}28.2 \\
( \pm 2.1)\end{array}$ & $\begin{array}{c}0.8 \\
( \pm 0.1)\end{array}$ \\
\hline
\end{tabular}

Horizons: ak, anthric; ak/lv, intermediary; um, umbric; ab, albic; um/ab, intermediary; lv, luvic; fg, fragic; st, stagnic; cc, calcic. Physical properties: $\rho_{\mathrm{s}}$, particle density; $\rho_{\mathrm{d}}$, bulk density dry; $\mathrm{P}_{\mathrm{T}}$, total porosity; $\mathrm{P}_{\mathrm{N}}$, coarse pores; $\mathrm{P}_{\mathrm{C}}$, capillary pores; $\mathrm{P}_{\mathrm{S}}$, semi-capillary pores. Texture evaluated in triangle diagram: ssh, silty-loam; sp, sandy-loam; sh, loamy; ssi, silty-clay-loam; si, clay-loam; spi, sandy clay loam; ts, silty clay; ti, clay; tp, sandy clay. Texture by Novák based on percentage of particles $<0.01$ mm: H, loamy; PH, sandy-loam; IH, clay-loam; HP, loamy-sand. 
Table 2. Mean ( \pm standard deviation) soil hydro-physical properties in profiles

\begin{tabular}{|c|c|c|c|c|c|c|c|c|c|}
\hline Tree species & Horizon & $\begin{array}{c}\text { Depth } \\
\text { (m) }\end{array}$ & $\Theta_{\mathrm{RC}}$ & $\Theta_{\mathrm{CMC}}$ & Wv & $\begin{array}{c}\Theta v \\
(\%)\end{array}$ & $\Theta p$ & $\mathrm{~V}_{\mathrm{AM}}$ & $\mathrm{V}_{\mathrm{A}}$ \\
\hline \multirow{3}{*}{$\begin{array}{l}\text { Lawson } \\
\text { cypress }\end{array}$} & ak & $0-30$ & $\begin{array}{c}31.7 \\
( \pm 1.6)\end{array}$ & $\begin{array}{c}34.7 \\
( \pm 1.2)\end{array}$ & $\begin{array}{c}13.9 \\
( \pm 4.7)\end{array}$ & $\begin{array}{c}17.1 \\
( \pm 1.2)\end{array}$ & $\begin{array}{c}14.6 \\
( \pm 2.2)\end{array}$ & $\begin{array}{c}25.4 \\
( \pm 7.7)\end{array}$ & $\begin{array}{c}14.1 \\
( \pm 5.3)\end{array}$ \\
\hline & lv & $30-70$ & $\begin{array}{c}32.6 \\
( \pm 1.0)\end{array}$ & $\begin{array}{c}34.5 \\
( \pm 0.7)\end{array}$ & $\begin{array}{c}15.6 \\
( \pm 2.4)\end{array}$ & $\begin{array}{c}18.6 \\
( \pm 0.6)\end{array}$ & $\begin{array}{c}13.9 \\
( \pm 1.0)\end{array}$ & $\begin{array}{c}19.3 \\
( \pm 2.3)\end{array}$ & $\begin{array}{c}7.5 \\
( \pm 2.0)\end{array}$ \\
\hline & $\mathrm{cc}$ & $70-80$ & $\begin{array}{c}33.3 \\
( \pm 0.9)\end{array}$ & $\begin{array}{c}34.6 \\
( \pm 1.1)\end{array}$ & $\begin{array}{c}15.1 \\
( \pm 0.5)\end{array}$ & $\begin{array}{c}19.9 \\
( \pm 0.3)\end{array}$ & $\begin{array}{c}13.4 \\
( \pm 0.2)\end{array}$ & $\begin{array}{c}17.4 \\
( \pm 1.8)\end{array}$ & $\begin{array}{c}5.0 \\
( \pm 1.7)\end{array}$ \\
\hline \multirow{4}{*}{ Tulip trees } & um & $0-20$ & $\begin{array}{c}35.6 \\
( \pm 1.9)\end{array}$ & $\begin{array}{c}39.5 \\
( \pm 2.0)\end{array}$ & $\begin{array}{c}20.8 \\
( \pm 2.8)\end{array}$ & $\begin{array}{c}14.8 \\
( \pm 1.0)\end{array}$ & $\begin{array}{c}20.8 \\
( \pm 1.5)\end{array}$ & $\begin{array}{c}24.2 \\
( \pm 2.8)\end{array}$ & $\begin{array}{c}16.5 \\
( \pm 3.0)\end{array}$ \\
\hline & $a b$ & $20-50$ & $\begin{array}{c}32.9 \\
( \pm 2.1)\end{array}$ & $\begin{array}{c}36.2 \\
( \pm 2.7)\end{array}$ & $\begin{array}{c}25.3 \\
( \pm 2.8)\end{array}$ & $\begin{array}{c}15.0 \\
( \pm 0.9)\end{array}$ & $\begin{array}{c}17.8 \\
( \pm 2.1)\end{array}$ & $\begin{array}{c}18.5 \\
( \pm 2.8)\end{array}$ & $\begin{array}{c}13.4 \\
( \pm 1.9)\end{array}$ \\
\hline & lv & $50-70$ & $\begin{array}{c}29.4 \\
( \pm 0.8)\end{array}$ & $\begin{array}{c}33.9 \\
( \pm 1.1)\end{array}$ & $\begin{array}{c}26.1 \\
( \pm 2.3)\end{array}$ & $\begin{array}{c}15.4 \\
( \pm 0.9)\end{array}$ & $\begin{array}{c}14.1 \\
( \pm 1.4)\end{array}$ & $\begin{array}{c}15.2 \\
( \pm 3.7)\end{array}$ & $\begin{array}{c}10.3 \\
( \pm 3.3)\end{array}$ \\
\hline & st & $70-90$ & $\begin{array}{c}31.5 \\
( \pm 0.9)\end{array}$ & $\begin{array}{c}32.5 \\
( \pm 0.6)\end{array}$ & $\begin{array}{c}29.9 \\
( \pm 4.7)\end{array}$ & $\begin{array}{c}19.0 \\
( \pm 0.9)\end{array}$ & $\begin{array}{c}12.5 \\
( \pm 1.5)\end{array}$ & $\begin{array}{c}8.7 \\
( \pm 2.9)\end{array}$ & $\begin{array}{c}7.9 \\
( \pm 1.3)\end{array}$ \\
\hline \multirow{3}{*}{$\begin{array}{l}\text { Chinese } \\
\text { junipers }\end{array}$} & ak & $0-20$ & $\begin{array}{c}23.1 \\
( \pm 2.7)\end{array}$ & $\begin{array}{c}23.8 \\
( \pm 2.9)\end{array}$ & $\begin{array}{c}3.6 \\
( \pm 3.8)\end{array}$ & $\begin{array}{c}17.0 \\
( \pm 1.7)\end{array}$ & $\begin{array}{c}6.1 \\
( \pm 2.3)\end{array}$ & $\begin{array}{c}38.1 \\
( \pm 9.0)\end{array}$ & $\begin{array}{c}32.8 \\
( \pm 10.1)\end{array}$ \\
\hline & lv & $20-30$ & $\begin{array}{c}31.0 \\
( \pm 5.0)\end{array}$ & $\begin{array}{c}31.7 \\
( \pm 5.4)\end{array}$ & $\begin{array}{c}11.6 \\
( \pm 2.5)\end{array}$ & $\begin{array}{c}21.5 \\
( \pm 0.6)\end{array}$ & $\begin{array}{c}9.5 \\
( \pm 4.5)\end{array}$ & $\begin{array}{c}22.5 \\
( \pm 2.4)\end{array}$ & $\begin{array}{c}14.3 \\
( \pm 4.4)\end{array}$ \\
\hline & st & $30-80$ & $\begin{array}{c}36.6 \\
( \pm 2.1)\end{array}$ & $\begin{array}{c}36.9 \\
( \pm 2.3)\end{array}$ & $\begin{array}{c}19.9 \\
( \pm 1.0)\end{array}$ & $\begin{array}{c}25.4 \\
( \pm 1.7)\end{array}$ & $\begin{array}{c}11.5 \\
( \pm 3.7)\end{array}$ & $\begin{array}{c}10.3 \\
( \pm 1.9)\end{array}$ & $\begin{array}{c}2.6 \\
( \pm 1.2)\end{array}$ \\
\hline \multirow{3}{*}{$\begin{array}{c}\text { Oriental } \\
\text { thujas }\end{array}$} & ak & $0-20$ & $\begin{array}{c}32.3 \\
( \pm 0.6)\end{array}$ & $\begin{array}{c}35.0 \\
( \pm 1.5)\end{array}$ & $\begin{array}{c}10.1 \\
( \pm 5.9)\end{array}$ & $\begin{array}{c}19.5 \\
( \pm 4.8)\end{array}$ & $\begin{array}{c}12.8 \\
( \pm 5.4)\end{array}$ & $\begin{array}{c}27.1 \\
( \pm 4.5)\end{array}$ & $\begin{array}{c}15.1 \\
( \pm 4.9)\end{array}$ \\
\hline & $\mathrm{ak} / \mathrm{lv}$ & $20-40$ & $\begin{array}{c}32.1 \\
( \pm 1.8)\end{array}$ & $\begin{array}{c}35.8 \\
( \pm 0.4)\end{array}$ & $\begin{array}{c}7.2 \\
( \pm 6.0)\end{array}$ & $\begin{array}{c}19.7 \\
( \pm 2.2)\end{array}$ & $\begin{array}{c}12.2 \\
( \pm 3.7)\end{array}$ & $\begin{array}{c}26.0 \\
( \pm 5.4)\end{array}$ & $\begin{array}{c}9.4 \\
( \pm 3.3)\end{array}$ \\
\hline & $\mathrm{cc}$ & $40-80$ & $\begin{array}{c}31.3 \\
( \pm 0.2)\end{array}$ & $\begin{array}{c}35.0 \\
( \pm 0.7)\end{array}$ & $\begin{array}{c}11.1 \\
( \pm 0.6)\end{array}$ & $\begin{array}{c}12.9 \\
( \pm 0.4)\end{array}$ & $\begin{array}{c}18.3 \\
( \pm 0.3)\end{array}$ & $\begin{array}{c}29.0 \\
( \pm 1.5)\end{array}$ & $\begin{array}{c}10.9 \\
( \pm 1.6)\end{array}$ \\
\hline \multirow{4}{*}{$\begin{array}{l}\text { Western } \\
\text { redcedars }\end{array}$} & um & $0-10$ & $\begin{array}{c}25.7 \\
( \pm 1.8)\end{array}$ & $\begin{array}{c}27.1 \\
( \pm 1.8)\end{array}$ & $\begin{array}{c}11.8 \\
( \pm 2.4)\end{array}$ & $\begin{array}{c}16.0 \\
( \pm 1.3)\end{array}$ & $\begin{array}{c}9.7 \\
( \pm 3.1)\end{array}$ & $\begin{array}{c}30.6 \\
( \pm 4.5)\end{array}$ & $\begin{array}{c}27.4 \\
( \pm 1.4)\end{array}$ \\
\hline & um/ab & $10-22$ & $\begin{array}{c}31.0 \\
( \pm 4.0)\end{array}$ & $\begin{array}{c}36.0 \\
( \pm 4.4)\end{array}$ & $\begin{array}{c}15.9 \\
( \pm 5.1)\end{array}$ & $\begin{array}{c}17.1 \\
( \pm 0.4)\end{array}$ & $\begin{array}{c}13.9 \\
( \pm 3.9)\end{array}$ & $\begin{array}{c}24.3 \\
( \pm 4.8)\end{array}$ & $\begin{array}{c}14.8 \\
( \pm 4.6)\end{array}$ \\
\hline & $\mathrm{fg}$ & $22-50$ & $\begin{array}{c}30.6 \\
( \pm 2.7)\end{array}$ & $\begin{array}{c}36.8 \\
( \pm 3.1)\end{array}$ & $\begin{array}{c}16.9 \\
( \pm 2.2)\end{array}$ & $\begin{array}{c}15.3 \\
( \pm 2.1)\end{array}$ & $\begin{array}{c}15.3 \\
( \pm 3.5)\end{array}$ & $\begin{array}{c}27.6 \\
( \pm 3.9)\end{array}$ & $\begin{array}{c}19.8 \\
( \pm 2.7)\end{array}$ \\
\hline & st & $50-80$ & $\begin{array}{c}34.2 \\
( \pm 1.2)\end{array}$ & $\begin{array}{c}35.6 \\
( \pm 0.7)\end{array}$ & $\begin{array}{c}29.9 \\
( \pm 2.5)\end{array}$ & $\begin{array}{c}25.2 \\
( \pm 0.9)\end{array}$ & $\begin{array}{c}9.0 \\
( \pm 1.9)\end{array}$ & $\begin{array}{c}4.1 \\
( \pm 2.1)\end{array}$ & $\begin{array}{c}5.0 \\
( \pm 1.4)\end{array}$ \\
\hline \multirow{4}{*}{$\begin{array}{l}\text { Oriental } \\
\text { spruces }\end{array}$} & um & $0-20$ & $\begin{array}{c}27.3 \\
( \pm 3.5)\end{array}$ & $\begin{array}{c}29.3 \\
( \pm 4.0)\end{array}$ & $\begin{array}{c}11.4 \\
( \pm 3.6)\end{array}$ & $\begin{array}{c}12.7 \\
( \pm 0.5)\end{array}$ & $\begin{array}{c}14.6 \\
( \pm 3.9)\end{array}$ & $\begin{array}{c}35.1 \\
( \pm 6.1)\end{array}$ & $\begin{array}{c}27.9 \\
( \pm 6.0)\end{array}$ \\
\hline & lv & $20-40$ & $\begin{array}{c}28.5 \\
( \pm 0.7)\end{array}$ & $\begin{array}{c}32.1 \\
( \pm 0.7)\end{array}$ & $\begin{array}{c}19.4 \\
( \pm 0.9)\end{array}$ & $\begin{array}{c}14.7 \\
( \pm 1.8)\end{array}$ & $\begin{array}{c}13.8 \\
( \pm 1.4)\end{array}$ & $\begin{array}{c}22.3 \\
( \pm 2.3)\end{array}$ & $\begin{array}{c}15.3 \\
( \pm 2.3)\end{array}$ \\
\hline & st $_{1}$ & $40-62$ & $\begin{array}{c}30.6 \\
( \pm 2.1)\end{array}$ & $\begin{array}{c}32.4 \\
( \pm 1.9)\end{array}$ & $\begin{array}{c}20.9 \\
( \pm 3.9)\end{array}$ & $\begin{array}{c}20.3 \\
( \pm 1.4)\end{array}$ & $\begin{array}{c}10.3 \\
( \pm 2.8)\end{array}$ & $\begin{array}{c}13.9 \\
( \pm 2.5)\end{array}$ & $\begin{array}{c}9.0 \\
( \pm 2.0)\end{array}$ \\
\hline & $\mathrm{st}_{2}$ & $62-80$ & $\begin{array}{c}33.5 \\
( \pm 2.8)\end{array}$ & $\begin{array}{c}34.7 \\
( \pm 2.8)\end{array}$ & $\begin{array}{c}26.6 \\
( \pm 4.3)\end{array}$ & $\begin{array}{c}20.7 \\
( \pm 0.1)\end{array}$ & $\begin{array}{c}12.8 \\
( \pm 3.0)\end{array}$ & $\begin{array}{c}8.7 \\
( \pm 2.3)\end{array}$ & $\begin{array}{c}4.7 \\
( \pm 1.9)\end{array}$ \\
\hline \multirow{4}{*}{$\begin{array}{c}\text { Austrian } \\
\text { pines }\end{array}$} & um & $0-10$ & $\begin{array}{c}20.7 \\
( \pm 1.1)\end{array}$ & $\begin{array}{c}21.5 \\
( \pm 1.5)\end{array}$ & $\begin{array}{c}5.2 \\
( \pm 0.5)\end{array}$ & $\begin{array}{c}10.3 \\
( \pm 0.7)\end{array}$ & $\begin{array}{c}10.4 \\
( \pm 0.8)\end{array}$ & $\begin{array}{c}45.1 \\
( \pm 2.2)\end{array}$ & $\begin{array}{c}39.0 \\
( \pm 3.4)\end{array}$ \\
\hline & $a b$ & $10-38$ & $\begin{array}{c}23.0 \\
( \pm 2.9)\end{array}$ & $\begin{array}{c}25.9 \\
( \pm 3.0)\end{array}$ & $\begin{array}{c}14.9 \\
( \pm 1.7)\end{array}$ & $\begin{array}{c}8.0 \\
( \pm 0.4)\end{array}$ & $\begin{array}{c}15.0 \\
( \pm 2.8)\end{array}$ & $\begin{array}{c}30.9 \\
( \pm 2.7)\end{array}$ & $\begin{array}{c}21.6 \\
( \pm 3.5)\end{array}$ \\
\hline & fg & $38-70$ & $\begin{array}{c}15.6 \\
( \pm 2.6)\end{array}$ & $\begin{array}{c}17.5 \\
( \pm 2.8)\end{array}$ & $\begin{array}{c}13.8 \\
( \pm 5.1)\end{array}$ & $\begin{array}{c}7.8 \\
( \pm 1.2)\end{array}$ & $\begin{array}{c}7.7 \\
( \pm 3.7)\end{array}$ & $\begin{array}{c}25.7 \\
( \pm 2.8)\end{array}$ & $\begin{array}{c}21.6 \\
( \pm 2.7)\end{array}$ \\
\hline & st & $70-90$ & $\begin{array}{c}28.2 \\
( \pm 2.1) \\
\end{array}$ & $\begin{array}{c}28.7 \\
( \pm 2.2) \\
\end{array}$ & $\begin{array}{c}27.8 \\
( \pm 0.9)\end{array}$ & $\begin{array}{c}15.6 \\
( \pm 0.3) \\
\end{array}$ & $\begin{array}{c}12.6 \\
( \pm 0.5) \\
\end{array}$ & $\begin{array}{c}9.1 \\
( \pm 1.5) \\
\end{array}$ & $\begin{array}{c}6.0 \\
( \pm 1.4) \\
\end{array}$ \\
\hline
\end{tabular}

Horizons: ak, anthric; ak/lv, intermediary; um, umbric; ab, albic; um/ab, intermediary; lv, luvic; fg, fragic; st, stagnic; cc, calcic. Hydro-physical properties: $\theta_{\mathrm{RC}}$, retention water capacity; $\theta_{\mathrm{CMC}}$, maximum capillary water capacity; Wv, available water supply; $\theta_{\mathrm{V}}$, wilting point; $\theta_{\mathrm{P}}$, available water capacity; $\mathrm{V}_{\mathrm{AM}}$, actual air volume; $\mathrm{V}_{\mathrm{A}}$, air-filled porosity. 
BRADY and WeIL (1999) reported that macropores volume representing less than $10 \%$ of the total soil volume can cause significant inhibition of the microbial activity and plant growth in most soils. The results determined in the soil of the Arboretum showed that the coarse pores were extremely scarce, dependent on the species: from the depth of $0.2 \mathrm{~m}$ (under oriental thujas), from $0.3 \mathrm{~m}$ (under Lawson cypress and Chinese junipers), from $0.4 \mathrm{~m}$ and $0.5 \mathrm{~m}$ (under oriental spruces and western redcedars) (Table 1, Fig. 1), therefore roots ranging deeper may suffer from oxygen shortage. On the other hand, the main volume of tree roots extends to the depth of 0.4-0.5 m (Š́́́LY, 1978).

In all the studied profiles, soil aeration $\left(\mathrm{V}_{\mathrm{AM}}\right)$ as well as air-filled porosity decreased with depth, although significant reduction of air content was found in all st horizons and also in lv horizon under Lawson cypress and intermediary ak/lv horizon under oriental thujas (Table 2). LHotský et al. (1984) presented value of $10 \%$ as the limit $\mathrm{V}_{\mathrm{A}}$. Decreased $\mathrm{V}_{\mathrm{A}}$ and impeded aeration can result from the mechanical compaction of the soil, poor drainage and water logging, and heavy soil texture (HoušKová, 2002; WAll and HeisKanen, 2009; PolLÁKová et al., 2016). Generally, under poor soil aeration even anaerobic or waterlogged conditions, existing roots stop growing and new roots do not penetrate into the waterlogged ground. Chemical reactions running in the absence of oxygen in the existing roots, can produce damaging toxins causing the roots dying. Paradoxically, waterlogging can cause problems of drought in summer through limited root expansion. Fluctuating water levels can be the worst as they may lead to continually wasted investment in new roots (HUXLEY et al., 1992).

However, based on the annual surveys of plants abiotic damages in the Arboretum, it can be stated that studied trees had not suffered by lack of soil aeration. On the contrary, some tree species, such as Japanese cedar, tend to suffer with lack of soil moisture, manifested as increased litterfall and weak growth (PoLLÁKOvá et al., 2016), mainly during summer season, which is normally hot and dry (HRUBík et al., 2011). Moreover, within the period 1971-2011, the mean annual air temperature and mean spring air temperature in the Arboretum increased by $1.41{ }^{\circ} \mathrm{C}$ or $1.06{ }^{\circ} \mathrm{C}$, respectively. This temperature increase affected the onset of phenophases and the length of growing season. The beginning of growing season was advanced by 19 days for European (1990-2011) and 9 days for Asian taxa (1991-2008) (BARTA and HoŤKA, 2013). Logically, longer growing season is related to increased demands on water for transpiration.

Physical and hydro-physical properties of studied soil profiles and their meeting the demands of introduced tree species

Beside Austrian pines, Chinese junipers, oriental thujas and oriental spruces, the other tree species investigated in this study had high requirements on soil moisture. Even western redcedars are able to grow in swampy areas and on river banks (HUXLEY et al., 1992; HoRÁČEK, 2007; Hieke, 2008; BeCK, 2017; Van HaVerbeKe, 2017; Minore, 2017; ZoBel, 2017). Nevertheless, all the studied trees were more than 40 years old, and according to Š́́LY (1978), young trees barely tolerate the lack of water, while older ones are more resilient. In addition, during juvenescence of studied trees, the climate in the region was more favourable. However, particularly in the last two decades, many extremely dry years occurred (IšToŇA and PAVLENDA, 2011). Soil drought accompanied by dry air can affect substantially the transpiration and consequently the soil water dynamics in the root zone (MATEJKA et al., 2009). We also investigated, whether the studied trees were planted in a climatically appropriate location, according to the climatic regionization (SuPUKA et al., 1991). We found that the Austrian pines, oriental thujas, Chinese junipers and Lawson cypresses profit from the warm and dry climate of the Arboretum locality, while western redcedars, oriental spruces and tulip trees have limited conditions in the given climatic area.

Consider Lawson cypresses, succeed in full sun on moist, well drained soils, with high rainfall and humidity, but can survive on dry alkaline soils (HUXLEY et al., 1992; HiEke, 2008). The soil with Lawson cypresses was from the depth of $0.3 \mathrm{~m}$ characterized by low percentage of coarse pores, and air-filled porosity (Tables 1-2) what was a prerequisite of reduced roots breathing. On the other side, Lawson cypresses have no taproot but produce vertical sinkers from the dense network of fibrous horizontal root system (ZOBEL, 2017). Therefore, they cannot suffer with shortage of oxygen in the studied soil. High percentage (32.6-33.3\%) of $\mathrm{P}_{\mathrm{C}}$ retaining rainwater and medium texture ensured that the values of wilting point $\left(\theta_{\mathrm{V}}\right)$ are relatively low $(18.6-19.9 \%)$, whereas available water capacity $\left(\theta_{\mathrm{p}}\right)$ high, mainly in $\mathrm{lv}$ and cc horizons (13.4-13.9\%) and Lawson cypresses flourish, despite preference for moist soils (Tables 1-2).

Also tulip trees prefer deep, rich, rather moist soils (HorÁČEK, 2007). Soil physical properties strongly overshadow chemical properties in determining distribution and growth of tulip trees. They grow naturally and well in soils moderately moist, well drained, and loose textured; they rarely do well in very wet or very dry areas. Exceptionally good growth has been observed on alluvial soils bordering streams, on loam soils of mountain coves, on talus slopes below cliffs and bluffs, and on well-watered, gravelly soils. They have a rapidly growing and deeply penetrating juvenile taproot, as well as many strongly developed and widespreading lateral roots (BECK, 2017). The stand of tulip trees in the Arboretum was located in a terrain depression, at a foot of a gentle slope, 50 meters from a pond. The studied profile has $10 \%$ stoniness, $31-55 \%$ sand content, $8-31 \%$ content of clay, ensuring that values 
of $\theta_{\mathrm{p}}$ are high (Tables 1-2). Moreover, 29.4-35.6\% of $\mathrm{P}_{\mathrm{C}}$ is not only able to maintain enough rainwater, but also elevate groundwater by capillary raise. Likewise, tulip trees on their stand meet good soil conditions for growth, therefore well thrive.

Chinese junipers need for optimal growth welldrained soil, or they will decline from root rot. They are quite drought-tolerant and succeed also in dry areas (HuXley et al., 1992; Hieke, 2008). The soil physical properties on Chinese junipers site in the Arboretum, do not provide good conditions for quick drainage, since from $0.3 \mathrm{~m}$ the coarse porosity is only $2.2 \%$, capillary porosity $36.6 \%$ (even $\mathrm{P}_{\mathrm{C}}$ represents up to $93 \%$ of $\mathrm{P}_{\mathrm{T}}$ ), and clay content is $44.8 \%$. The low value of $\mathrm{V}_{\mathrm{A}}(2.6 \%)$ does not guarantee sufficient air exchange at depth under $0.3 \mathrm{~m}$ (Tables 1-2). Therefore, on the base of results of physical soil properties, this site reveals a high probability that the Chinese junipers would suffer with a root rot during late autumn, winter and early spring. However, up to now there were not found any roots damages.

Another studied dry tolerant tree species were oriental thujas, best growing on dry, freely drained sites, in wild grow mainly on steep rocky valley sides, often on cliffs in dry areas of Western China (HuXLEY et al., 1992). The soil in the Arboretum, where oriental thujas are grown is characterized by high content of silt (51.4-54.9\%) in the whole profile, including soil-forming substrate, which is carbonate loess. Such high amount of silt influences formation of favourable porosity (ŠARAPATKA, 2014), and thus quick drainage, but also water maintenance in capillary pores (Table 1 ). The evidence of suitable conditions is a healthy, 50 years old, dense growth of oriental thujas.

Western redcedars demand high soil moisture, and grow well in ravines and on poorly drained soils in depressions (HiEke, 2008). Their tap-roots are poorly defined or nonexistent, but fine roots develop a profuse, dense network. Shallower root systems are more frequent either in soils with high bulk density or on wet sites than on deep, moderately dry soils (MINORE, 2017). Soil under redcedars in the Arboretum is characterized by $5 \%$ stoniness, $35-44.4 \%$ content of sand, which results in a $13.8-28.3 \%$ coarse porosity up to the depth of $0.5 \mathrm{~m}$, a prerequisite for quick drainage of rainwater. On the contrary, the compacted, enriched by clay st horizon is characterized by an extremely low coarse porosity (4.7\%) and therefore reduced water drainage, respectively by a high retention of rainwater. However, the st horizon, despite high retention water capacity $(34.2 \%)$, has a low value of available water capacity $(9.0 \%)$, because the high clay content $(47.3 \%)$ considerably increasing values of wilting point $(25.2 \%)$. Thus the substantial amount of water in st horizon is physiologically dead, unavailable to western redcedars demanding high soil moisture (Tables 1-2). Nevertheless, the soil-climate conditions described in this study are exceptionally favourable for western redcedars, and the species is spreading spontaneously over this site.

Oriental spruces should be grown on well-drained soil, and they tolerate infertile, even rocky soils, however, only in locations, where winters are not extremely dry (HuXley et al., 1992; GILMAN and WATSON, 1994). Stagnic horizons $\left(\mathrm{st}_{1}, \mathrm{st}_{2}\right)$ enriched by clay (38.4 and $43.2 \%)$ slow down the proper drainage of rainwater $\left(\mathrm{P}_{\mathrm{N}}\right.$ $4.1-8.2 \%$ ). However, $10 \%$ and $20 \%$ content of gravel in these horizons may increase their drainage ability. Good health status and large height of trees give evidence for appropriate adaptation of oriental spruces on their site in the Arboretum (Tables 1-2).

Among all the examined tree species, the Austrian pines are the least demanding on soil properties. They can grow on rocky, often poor, calcareous, even pure limestone, sandy, dry areas (KovÁč et al., 2005; HiEke, 2008). Austrian pines are deep laterally rooted, and therefore, perform best in deep soils. On good sites they grow fast and straight, up to $40 \mathrm{~m}$ (VAN HAVERBEKE, 2017). The Austrian pines in the Arboretum were planted on a hill-top, to serve as a protective strip for other introduced trees, mainly against the wind and also due to bad soil properties on the site. The soil on site of Austrian pines is characterized by $53.6-64.7 \%$ of sand and 10-30\% stoniness. STEINHÜBEL (1957) reported, that gravel is usually rounded, dominated by quartz, less quartzite, and occasionally andesite and other rocks building the Tribeč Mts, from which the gravel originates. On this material the loess was accumulated and over time decarbonated due to soil-forming processes. Soil formed on such soil-forming substrate is well drained. On the other side, $38.4 \%$ of clay in st horizon in depth of $0.7 \mathrm{~m}$ contributes to the retention of $28.2 \%$ water, from which nearly half is available $\left(\theta_{\mathrm{P}} 12.6 \%\right)$ to trees roots (Tables 1-2), what satisfies Austrian pines demands, and grow up to $20 \mathrm{~m}$.

According to this study results, not all the tree species introduced into the Arboretum Mlyňany meet there optimal conditions for their growth. Nevertheless, they have acclimatized well in soil-climatic conditions described in this study.

\section{Conclusions}

Currently only few scientific works consider the relationships between introduced tree species and properties of soils, on which they are grown. The results presented in this study enrich the knowledge about possibilities of growing selected introduced tree species on soils with similar physical properties in temperate climate of Central Europe.

The results of this study showed, that all stagnic horizons exceeded limit values of total porosity and bulk density, hence were compacted.

Based on the literature knowledge about soil and climatic requirements of the examined trees we may conclude that soil conditions of their sites in Arboretum 
Mlyňany comply to: Lawson cypress, tulip trees, oriental thuja trees, Austrian pines. However, physical properties in the profiles under oriental spruces and Chinese junipers do not permit rapid drainage of water, and this is unfavourable for their healthy development, because they usually suffer from root rot in excessively wet soil. On the contrary, Western redcedar demands high soil moisture, but the soil profile under redcedars in the Arboretum is characterized by a high coarse porosity up to the depth of $0.5 \mathrm{~m}$, what is a prerequisite of fast rainwater drainage.

Despite not all the tree species introduced into the Arboretum Mlyňany meet there optimal conditions for their growth, none of them suffered from lack of soil aeration or moisture and all the species seem acclimatized well in the soil-climatic conditions of the Arboretum Mlyňany.

\section{Acknowledgement}

The authors want to acknowledge the financial support by the Cultural and Educational Grant Agency of the Ministry of Education, Science, Research and Sport of the Slovak Republic (KEGA 014 SPU - 4/2016). We thank to Kristína Malachovská for improving the English.

\section{References}

BARTA, M., HoŤKA, P., 2013. Variability in the growing season of selected European and East-Asian woody species in relation to air temperature changes. Folia Oecologica, 40: 1-10.

BECK, D.E., 2017. Liriodendron tulipifera L., YellowPoplar, Magnoliaceae - Magnolia family [cit. 20171-16]. https://www.na.fs.fed.us/pubs/silvics_manual/volume_2/liriodendron/tulipifera.htm

BRADY, N.G., WeIL, R.R., 1999. The nature and properties of soils. Upper Saddle River, New Jersey: Prentice Hall. 881 p.

CIFrA, J., 1958. Stručná charakteristika pôdnych pomerov Arboréta Mlyňany [Short characteristic of soil conditions in the Arboretum Mlyňany]. In BENČǍ̆, F. (ed.). Prírodné podmienky Arboréta Mlyňany. Bratislava: Vydavatel'stvo Slovenskej akadémie vied, p. 79-96.

Dexter, A.R., 2004. Soil physical quality. Part I. Theory, effects of soil texture, density, and organic mater, and effects on root growth. Geoderma, 120: 201-214.

ELIÁš, P., 2011. Introdukcia nepôvodných druhov ako prvý krok $\mathrm{k}$ invázii drevín [Introduction of alien species as the first step of woody plants invasion]. In BARTA, M., KonôPKová, J. (eds). Dendrologické dni v Arboréte Mlyňany SAV 2011. Aktuálne otázky štúdia introdukovaných drevín. Zborník referátov $z$ konferencie. Vieska nad Žitavou: Arborétum Mlyňany SAV, p. 29-41.

Frey, B., Kremer, J., RÜDt, A., Sciacca, S., Matthies, D., LÜSCHER, P., 2009. Compaction of forest soils with heavy logging machinery affects soil bacterial community structure. European Journal of Soil Biology, 45: 312-320.

Gilman, E.F., Watson, D.G., 1994. Juniperus chinensis 'Torulosa': 'Torulosa' Juniper. Fact Sheet ST-452 (Environmental Horticulture Department, Florida Cooperative Extension Service, Institute of Food and Agricultural Sciences, University of Florida) [cit. 2017-2-12]. http://hort.ifas.ufl.edu/database/ documents/pdf/tree fact sheets/picoria.pdf

HIEKE, K., 2008. Encyklopedie jehličnatých stromů a keř̀ [Encyclopedia of coniferous trees and shrubs]. Brno: Computer press. 246 p.

HorÁČEK, P., 2007. Encyklopedie listnatých stromů a keřu [Encyclopedia of deciduous trees and shrubs]. Brno: Computer press. 747 p.

Hosseinia, F., Mosaddeghia, M.R., Hajabbasia, M.A., SabZaLian, M.R., 2016. Role of fungal endophyte of tall fescue (Epichloë coenophiala) on water availability, wilting point and integral energy in texturally-different soils. Agricultural Water Management, 163: 197-211.

Hoťka, P., Barta, M., Bibeñ, T., 2013. Study of the richest gene pool of trees and shrubs in Slovakia, in the Mlyňany Arboretum SAS. Folia Oecologica, 40: 181-187.

HoušKová, B., 2002. Assessment of the state of soil compaction in Slovakia. In PagliaI, M., Jones, R. (eds). Sustainable land management - environmental protection. A soil physical approach. Advances in geoecology, 35. Reiskirchen: Catena, p. 379-386.

HrivŇÁKová, K., MaKovníKová, J., BARANČíková, G., BezÁk, P., Bezáková, Z., Dodok, R., Grečo, V., Chlpík, J., Kobza, J., LišTuaK, M., Mališ, J., Píš, V., Schlosserová, J., Slávik, O., STyK, J., Širáñ, M., 2011. Jednotné pracovné postupy rozborov pôd [Uniform methods of soil analyses]. Bratislava: Výskumný ústav pôdoznalectva a ochrany pôdy, 2011. $136 \mathrm{p}$.

Hrubík, P., HoŤKa, P., Fogadová, K., Kuba, J., 2011. Klimatické podmienky Arboréta Mlyňany SAV vo Vieske nad Žitavou za obdobie 1971-2011 [Climate conditions of the Arboretum Mlyňany in Vieska nad Žitavou for 1971-2011]. In BARTA, M., KoNôPKOvÁ, J. Dendrologické dni v Arboréte Mlyn̆any SAV 2011. Aktuálne otázky štúdia introdukovaných drevín. Zbornik referátov z konferencie. Vieska nad Žitavou: Arborétum Mlyňany SAV, 2011, p. 66-73.

Huxley, A.J., Griffiths, M., LeVy, M., 1992. The new Royal Horticultural Society dictionary of gardening. London: Macmillan. 815 p.

IštoňA, J., PAVlenda, P., 2011. Monitoring of water storage in forest soils on PMP Čifáre in the years 1999-2009. Lesnicky Časopis - Forestry Journal, 57: $178-186$. 
Jourgholami, M., Khoramizadeh, A., Zenner, E.K., 2016. Effects of soil compaction on seedling morphology, growth, and architecture of chestnut-leaved oak (Quercus castaneifolia). iForest, 10: 145-153.

KovÁč, L., Kostúrová, N., Miklisová, D., 2005. Comparison of collembolan assemblages (Hexopoda, Collembola) of thermophilous oak woods and Pinus nigra plantations in the Slovak Karst (Slovakia). Pedobiologia, 49: 29-40.

Lhotský, J., VÁChal, J., Ehrich P., 1984. Soustava opatření k zúrodňovování zhutnělých půd [Measures for improving of compacted soils]. Metodiky pro zavádění výsledků výzkumu do zemědělské praxe. Praha: Ústav vědeckotechnických informací pro zemědělství. $39 \mathrm{p}$.

MatejKA, F., Střelcová, K., Hurtalová, T., Gömöryová, E., Ditmarová, L',, 2009. Seasonal changes in transpiration and soil water content in a spruce primeval forest during a dry period. In Střelcová, K., Mátyás, Cs., Kleidon, A., Lapin, M., Matejka, F., BlažEnec, M., ŠKvarenina, J., Holécy, J. (eds). Bioclimatology and natural hazards. Dordrecht: Springer, p. 197-206.

Mati, R., Kotorová, D., Gomboš, M., Kandra, B., 2011. Development of evapotranspiration and water supply of clay-loamy soil on the East Slovak Lowland. Agricultural Water Management, 98: 1133-1140.

Minore, D., 2017. Thuja plicata Donn ex D. Don, Western Redcedar, Cupressaceae - Cypress family. [cit. 2017-1-16]. https://www.na.fs.fed.us/spfo/ pubs/silvics_manual/Volume_1/thuja/plicata.htm

Pollákové, N., 2013. Soil subtypes classified in Nature Reserve Arboretum Mlyn̆any, Slovakia. Folia Oecologica, 40: 91-96.

Polláková, N., Šimanský, V., Kravka, M., JonczaK, J., 2016. Physical properties of soils under stands of introduced tree species in the Arboretum Mlyn̆any, Slovakia. Zprávy Lesnického Výzkumu, 61: 168174.

Safadoust, A., Feizeea, P., Mahboubib, A.A., GharaBaghib, B., Mosaddeghic, M.R., Ahrensda, B., 2014. Least limiting water range as affected by soil texture and cropping system. Agricultural Water Management, 136: 34-41.

SteInHüBel, G., 1957. Arborétum Mlyňany v minulosti a dnes [Arboretum Mlyňany in the past and in pre- sent]. Bratislava: Vydavatel'stvo Slovenskej akadémie vied. $145 \mathrm{p}$.

Supuka, J., BenČǍ̌, F., Bublinec, E., GÁPER, J., HruBík, P., Juhásová, G., MaglockÝ, Š., VReštiak, P. 1991. Ekologické princípy tvorby a ochrany zelene [Ecological principles of creation and protection of greenery]. Bratislava: Veda. 308 p.

Š́́LY, R., 1978. Pôda základ lesnej produkcie [Soil the base of forest productivity]. Bratislava: Príroda. $235 \mathrm{p}$.

ŠarapatKa, B., 2014. Pedologie a ochrana půdy [Pedology and soil protection]. Olomouc: Univerzita Palackého v Olomouci. 232 p.

TÁBOR, I., PAVlačKa, R., 1992. Arborétum Mlyňany [Arboretum Mlyňany]. Bratislava: Veda. 62 p.

TUŽInsKÝ, L., 2007. Bilancia vody v pôdach pod lesnými ekosystémami Slovenska - priestorová a časová variabilita vlhkosti pôdy [Water balance in soils in forest ecosystems in Slovakia - spatial and temporal variability in soil moisture content]. In STřElcová, K., ŠKvarenina, J., BlažEnec, M. (eds). Bioclimatology and natural hazards. International scientific conference. Proceedings. Zvolen - Pol'ana nad Detvou, Slovakia, 17-20 september 2007. Zvolen: Technical University. 8 p. 1 electronic optical disc (CD-ROM).

Van Haverbeke, D.F., 2017. Pinus nigra Arnold., European Black Pine, Pinaceae - Pine family. [cit. 2017-2-13]. https://www.na.fs.fed.us/spfo/pubs/silvics_manual/Volume_1/pinus/nigra.htm

Wall, A., Heiskanen, J., 2003. Water-retention characteristics and related physical properties of soil on afforested agricultural land in Finland. Forest Ecology and Management, 186: 21-32.

Wall, A., Heiskanen, J., 2009. Soil-water content and air-filled porosity affect height growth of Scots pine in afforested arable land in Finland. Forest Ecology and Management, 257: 1751-1756.

Zobel, D.B., 2017. Chamaecyparis lawsoniana (A. Murr.) Parl., Port-Orford-Cedar, Cupressaceae - Cypress family. [cit. 2017-2-23]. https://www. na.fs.fed.us/spfo/pubs/silvics_manual/Volume_1/ chamaecyparis/lawsoniana.htm

Received April 6, 2017 Accepted October 27, 2017 